\section{SMALL VOLUME STAGE 1B1 CERVICAL CANCER, IS RADICAL SURGERY STILL NECESSARY? A TEN YEAR FOLLOW UP}

${ }^{1} \mathrm{P}$ Korompelis*, ${ }^{1} \mathrm{~S}$ Rundle, ${ }^{1} \mathrm{~V}$ Cassar, ${ }^{2} \mathrm{~B}$ Biliatis, ${ }^{1} \mathrm{C}$ Ang, ${ }^{1} \mathrm{~A}$ Fisher, ${ }^{1} \mathrm{~N}$ Ratnavelu,

${ }^{1}$ A Kucukmetin. ' gateshead nhs trust, ngoc; ${ }^{2}$ poole nhs trust

10.1136/ijgc-2021-ESG0.91

Introduction/Background* Standard surgical treatment of FIGO stage 1B1 cervical cancer is open radical surgery. However, several reports have shown that for small tumours a more conservative approach can be oncologically safe whilst at the same time reducing the morbidity associated with radical hysterectomy. The objective of our study was to report updated survival following extended follow up of a previously reported cohort of patients who underwent conservative management of small-volume stage 1B1 disease.

Methodology All patients with FIGO stage 1B1 cancer and estimated tumour volume of less than $500 \mathrm{~mm} 3$ in a loop biopsy specimen treated in Norther Gynaecological Oncology Centre between January 2000 and December 2010, were included in the study, irrespective of other histological characteristics. Clinicopathological data alongside demographics were collated alongside detailed follow-up outcome in conjunction with primary care and death register.

Result(s)* 62 women underwent conservative management for small volume stage 1B1 disease. Median age at diagnosis was 35 years (range 25-67). 35 women (56.4\%) underwent fertility sparing conservative management with LLETZ while 27 women $(44.6 \%)$ underwent simple hysterectomy. Mean age of the LLETZ first group was 32 years (range 26-43) and 51\% were nulliparous. Overall, 92\% (57 out of 62) patients underwent BPND and one positive node was identified. LVSI was positive in 14 patients (22\%). Accurate survival data obtained $100 \%$ of the patients and no recurrence identified with median follow up of 11years (range 1-20). 2 patients died because of unrelated to cervical CA reasons (bowel CA 4 years post treatment and PE 1year post treatment).

Conclusion* The results of this study were initially published in 2012 with a median FU of 56 months (Biliatis et al. Gynaecologic Oncology 2012). Presenting long term FU data with median FU of 11 years we can strongly support that cervical loop biopsy or simple hysterectomy combined with negative pelvic lymphadenectomy for small-volume stage 1B1 cervical cancer offers excellent prognosis in terms of survival. Should these results be verified by further prospective studies, radical surgery for these women may be avoided.

\section{ABSTRACT WITHDRAWN}

\section{A REVIEW OF CERVICAL CANCER DIAGNOSED IN WOMEN OVER THE AGE OF 65}

S Baron*, S Kaushik. University Hospitals Sussex NHS Foundation Trust, Gynaecological Oncology, brighton, UK

\subsection{6/ijgc-2021-ESGO.92}

Introduction/Background*

Objectives To explore the incidence of cervix cancer following cessation of the UK cervical screening programme and compare this with the current UK screening programme guidelines.

Methodology 179 cases of cervical cancer diagnosed between 2016 and 2020 at University Hospitals Sussex were retrospectively reviewed. The screening history, grade, histology and stage of cancer were recorded.

Result(s)* Over a five-year period, 80 cases of cervical cancer were identified as being within the screened population. Of these $59(74 \%)$ were under 65 years and 21 (26\%) were over 65 years of age. An initial peak incidence was seen at 30-35 year age range, declining with further screening. Following cessation of screening, a secondary peak at 80-85 years was noted.

Of those diagnosed during screening (59.3\%) were FIGO IA1 to IB2, however, only $9.5 \%$ of the over 65 s were early stage. Similarly, $32.2 \%$ of those within screening age presented with a grade 1 cancer, with only $4.8 \%$ over 65 years being low grade. Histology in the under 65 s revealed $44.1 \%$ were squamous cell carcinoma and $45.8 \%$ were HPV-related adenocarcinoma. In the over $65 \mathrm{~s}$ this was $76.2 \%$ and $14.3 \%$ respectively.

Conclusion* Despite adherence to the screening programme, $25 \%$ of cervix cancer was diagnosed beyond screening age, approximately 16 years later. These patients were of more advanced stage and higher grade.

This preliminary exploration informs the need for a wider review of cervix cancer after the age of 65 and consideration of extension of the age of screening.

\section{RARE CASE OF INTESTINAL TYPE CERVICAL CANCER; CLINICAL PRESENTATION AND MANAGEMENT}

O Ali*. West Cumberland Hospital , OG, Whitehaven, UK

\subsection{6/ijgc-2021-ESG0.93}

Introduction/Background* Intestinal type cervical cancer is exceptionally rare and this a a c ase of 54 year presented with abnormal bleeding. The cancer is not normally picked up by the cervical screeing system. This is the story of multidisciplinary approach to managing such a case.

Methodology The case presented with abnormal bleeding with normal cervical screening history. She particularly complained of continuous mucus profuse per vaginal discharge. The ultrasound indicated fluid in the endometrium.The cervix looked abnormal and biopsies were taken. The histology indicated intestinal type adenocarcinoma. Her Ca19-9 was exceptionally high more than 2000iu, however CT scan did not indicate any pancreas or intestinal malignancy. The imaging including CT PET and MRI also indicated cervical tumor and one $7.5 \mathrm{~mm}$ left external iliac lymph node giving her stage $2 \mathrm{~B}$. The immunochemistry was positive for caudal tyoe homeobox2 (CDX20, Ca19-9, CEA, CK7, CK20 and it was negative for p16, p53 and estrogen. The histology demonstrated atypical signet ring and it was shown on the cervical, endometrial and vaginal biopsies.

Result(s)* The case was managed through multidisciplinary approach and recommended chemoradiation. She recieved brachy therapy and carboplatin and achieved radiological and clinical response. Subsequent follow up indocated some residual upper vaginal focus of tumor. She was offered sugery and she had radical hystrectomy and lymphadenopathy followed by full recovery. Two years follow indicated no recurrence clinically and radiologically. 
Conclusion* This case posed diagnostic and management dilemmas whether it is purely a cancer of the cervix or still representing an intestinal primary. The cancer is not HPV related so it can slip through the net of cervical screening and the main distinguished clinical feature was profuse mucus vaginal discahrge which greatly reduced after radiotherapy. The combination of neoadjuvant chemotherapy follwed by surgery was successful in this case. These enteric type cervical cancer represent a rare heterogenous group of cancers with variable presenations and prognosis.

\section{DOWNREGULATION OF LIMA1EXPRESSION INDUCES CANCER FEATURES IN NORMAL CERVICAL KERATINOCYTE HCK1T CELLS LEADING TO INCREASED MIGRATION AND INVASION}

${ }^{1}$ E Drakopoulou, ${ }^{1} E$ Kalafati*, ${ }^{1} \mathrm{AG}$ Vasilopoulou, ${ }^{1} \mathrm{~N}$ Anagnou, ${ }^{1 ; 2 \mathrm{~K}}$ Pappa. ${ }^{1}$ Biomedical Research Foundation of the Academy of Athens (BRFAA), Athens, Greece; ${ }^{2}$ University of Athens, First Department of Obstetrics and Gynecology, Athens, Greece

\subsection{6/ijgc-2021-ESGO.94}

Introduction/Background* Cervical cancer (CC) remains the fourth most common and lethal cancer type in women; therefore, a comprehensive understanding of the underlying mechanisms and development of novel diagnostic, prognostic and therapeutic approaches are critical steps for improving CC management. We have previously performed a detailed comparative proteomic analysis between $\mathrm{HeLa}, \mathrm{SiHa}$ and CD33A CC cell lines and the normal cervical keratinocytes HCK1T cells (Pappa et al, Oncol Rep. 42:1441, 2019) aiming to elucidate novel and potential therapeutic targets. Among hundreds of deregulated proteins between cancer and normal cell lines, LIMA1 or EPLIN, a protein decreased or lost in neoplasia, was statistically downregulated in all CC cell lines. Since LIMA1 role in CC is not fully elucidated yet, we aimed to downregulate its expression in HCK1T cells and assess the putative induction of cancer characteristics, such as increased proliferation, migration and invasion. Additionally, we studied the global m6A RNA methylation pattern in the presence and absence of LIMA1, aiming to highlight a potential role of epitranscriptomics in cancer onset and progression.

Methodology We downregulated LIMA1 expression in the HCK1T cell line using a lentiviral vector (LV) carrying a shRNA for either LIMA1 (shLIMA1) or no target (shNT) at a multiplicity of infection (MOI) 25. Following LIMA1 quantification both at protein and mRNA level, we assessed proliferation, invasion, migration and colony formation efficiency in shNT or shLIMA1 LV-transduced HCK1T cells. Proliferation was assessed using CCK8, while invasion and migration using Transwell $^{\circledR}$, with or without matrigel-coated inserts, respectively. Qualitative and quantitative assessment of m6A RNA methylation was done by $\mathrm{qPCR}$ and colorimetric EpiQuick ${ }^{\circledR}$ kit respectively.

Result(s)* Transduction with shLIMA1 LV led to a 98\% mean decrease of LIMA1 expression $(n=3)$, leading to a $126 \%$ $(\mathrm{p}=0.021, \mathrm{n}=3)$ and $103 \% \quad(\mathrm{p}=0.0091, \mathrm{n}=3)$ increase in migration and invasion respectively, while we did not observe any difference in cell proliferation and colony formation efficiency. Preliminary data regarding m6A RNA methylation point towards a slight increase following LIMA1 knockdown. Conclusion* LIMA1 may play an important role in cancer progression and metastasis and can set the grounds for novel and personalised therapy treatments for CC patients.

\section{6 THE DIFFERENTIAL EXPRESSION OF LIMA1/EPLIN IN NORMAL AND CERVICAL CANCER TISSUES}

${ }^{1}$ E Kalafati ${ }^{*},{ }^{1}$ E Drakopoulou, ${ }^{1} \mathrm{~N}$ Anagnou, ${ }^{1 ; 2}{ }^{2} \mathrm{~K}$ Pappa. ${ }^{1}$ Biomedical Research Foundation of the Academy of Athens (BRFAA), Athens, Greece; ${ }^{2}$ First Department of Obstetrics and Gynecology of the University of Athens, Athens, Greece

\subsection{6/ijgc-2021-ESGO.95}

Introduction/Background* LIMA1 or EPLIN is a cytoskeletonassociated protein involved in cytoskeleton regulation and dynamics, by inhibiting actin filament depolymerization and cross-linking filaments. The role of LIMA1 has been investigated in several malignant diseases including prostate, esophageal, breast and ovarian cancer. Its expression is often lost or aberrant in tumor cells leading to aggressive phenotypes. The aim of this study was to systematically assess the expression of LIMA1 in cervical cancer.

Methodology Immunohistochemical analysis using anti-EPLIN antibody was performed in 22 cases of cervical intraepithelial neoplasia, 14 cases of adenocarcinoma including one case with mucinous and one case with endometrioid adenocarcinoma, 5 cases of adenosquamous carcinoma, 25 cases of squamous cell carcinoma and 11 normal samples. The correlation of LIMA1 aberrant expression and disease progression was performed using the medical records of patients. Clinical samples of normal and tumor tissues were surgically excised, following an informed consent from all participants.

Result(s)* The levels of LIMA1 expression were low for healthy specimens, and limited to the cells of the basal membrane. LIMA1 expression showed a slight increase in the cases of intraepithelial neoplasia; however, it was quite low in cells displaying a HPV cytopathic effect (koilocytosis). On the contrary, the LIMA1 levels of both cytoplasmic and cellmembrane staining were significantly increased in the cases of adenocarcinoma and squamous cell carcinoma. Lastly, LIMA1 was highly expressed in undifferentiated, non-keratinizing, squamous cell carcinoma associated with poor prognosis.

Conclusion* Our results demonstrate that LIMA1 is differentially expressed not only in normal and cervical cancer tissues, but also in precancerous lesions and malignant cervical neoplasia, highlighting its role in cervical cancer progression and its potential exploitation as a prognostic factor.

\section{ABERRANT EXPRESSION OF ENZYMES REGULATING M6A RNA METHYLATION IN CERVICAL CANCER CELL LINES, AND THEIR IMPLICATION TO CERVICAL CANCER}

${ }^{1}$ E Drakopoulou, ${ }^{1} E$ Kalafati ${ }^{*},{ }^{1} \mathrm{AG}$ Vasilopoulou, ${ }^{1} \mathrm{~N}$ Anagnou, ${ }^{1 ; 2} \mathrm{~K}$ Pappa. ${ }^{1}$ Biomedical Research Foundation of the Academy of Athens (BRFAA), Athens, Greece; ${ }^{2}$ First Department of Obstetrics and Gynecology of the University of Athens, Athens, Greece

\subsection{6/ijgc-2021-ESG0.96}

Introduction/Background* RNA modifications play a key role in the regulation of gene expression. Among them, N6methyladenosine (m6A) is the most prevalent mRNA modification. m6A methylation modulates the gene expression by influencing numerous aspects of mRNA metabolism, including pre-mRNA processing, nuclear export, and translation. Many studies have demonstrated the importance of m6Amediated post-transcriptional gene regulation in numerous physiological and pathophysiological processes, especially in 